Projets

de paysage

\section{Projets de paysage}

Revue scientifique sur la conception et l'aménagement de l'espace

$15 \mid 2016$

L'observation et les observatoires de paysage

\title{
Focus sur les Observatoires photographiques du paysage (OPP) réalisés par le Conservatoire régional d'espaces naturels (CREN) de Poitou- Charentes
}

A Focus on Photographic Observatories of the Landscape Conducted by the Conservatoire régional d'espaces naturels (Regional Conservatory of Natural Spaces) of the region of Poitou-Charentes

Patrick Guédon

\section{(2) OpenEdition}

\section{Édition électronique}

URL : http://journals.openedition.org/paysage/6809

DOI : 10.4000/paysage.6809

ISSN : 1969-6124

\section{Éditeur :}

École nationale supérieure du paysage de Versailles-Marseille, Institut national des sciences appliquées Centre Val de Loire - École de la nature et du paysage, École nationale supérieure d'architecture et de paysage de Bordeaux, École nationale supérieure d'architecture et de paysage de Lille, Agrocampus Angers

\section{Référence électronique}

Patrick Guédon, « Focus sur les Observatoires photographiques du paysage (OPP) réalisés par le Conservatoire régional d'espaces naturels (CREN) de Poitou-Charentes », Projets de paysage [En ligne], 15 | 2016, mis en ligne le 31 décembre 2016, consulté le 13 juin 2020. URL : http:// journals.openedition.org/paysage/6809; DOI : https://doi.org/10.4000/paysage.6809

Ce document a été généré automatiquement le 13 juin 2020

Projets de paysage 


\title{
Focus sur les Observatoires
} photographiques du paysage (OPP) réalisés par le Conservatoire régional d'espaces naturels (CREN) de Poitou-Charentes

\author{
A Focus on Photographic Observatories of the Landscape Conducted by the \\ Conservatoire régional d'espaces naturels (Regional Conservatory of Natural \\ Spaces) of the region of Poitou-Charentes
}

Patrick Guédon

1 Le principe de confrontation de clichés de paysage, effectués dans des conditions de prises de vue similaires et à des moments distincts, a été développé à la fin des années 1990 par le ministère chargé de l'Environnement. Ce procédé permet d'évaluer au fil du temps les effets de la politique de l'État en matière d'environnement et de paysage.

2 En 1997, la direction de la Nature et des Paysages du ministère fait le point sur l'intérêt, la nature et les techniques de mise en place d'observatoires photographiques du paysage sur le territoire français dans Séquences Paysages, une revue de l'Observatoire photographique du paysage $\left(n^{\circ} 1\right)$ (ministère de l'environnement - HAZAN) :

3 «Les Français qui aiment leurs paysages, parfois passionnément, sont inquiets de leur devenir. Ceci les amène à se réfugier dans des attitudes trop souvent passéistes. Le travail de l'observatoire photographique du paysage va permettre une meilleure appréciation des problèmes...

4 La mise en place d'un système de veille photographique permet de faire ressortir sans délai les altérations du paysage, mais aussi certains progrès ou améliorations. Elle participe au suivi des territoires dont nous avons la charge... En associant l'approche sensible et l'approche scientifique, la mise en place de cet Observatoire photographique contribue à l'émergence d'une culture moderne de l'environnement. » 
5 Aujourd'hui, l'outil «Observatoire photographique national du paysage (OPNP)» comptabilise 20 itinéraires $^{1}$ dont la production est librement accessible sur Internet. Parallèlement à cette démarche nationale, une soixantaine d'observatoires photographiques du paysage (OPP) sont portés par des acteurs locaux.

\section{Pourquoi utiliser un tel outil dans la gestion des espaces naturels?}

6 Le Conservatoire régional d'espaces naturels (CREN) de Poitou-Charentes a pour mission « la sauvegarde, la protection, la mise en valeur et l'étude des sites, milieux et paysages naturels de Poitou-Charentes qui représentent un intérêt écologique, floristique, faunistique, biologique, géologique et paysager remarquable et de tous sites à valeur écologique potentielle ».

7 Le protocole du Conservatoire s'inspire du protocole national. Le CREN a développé cet outil pour répondre à des besoins spécifiques: observer et évaluer l'évolution des espaces d'intervention en maîtrise foncière ou d'usage, sous l'angle scientifique et paysager.

8 Ainsi, lorsqu'un site nécessite d'être équipé d'observatoires photographiques du paysage, l'antenne «paysage " propose une préimplantation de l'outil en confrontant des données cartographiques à une visite préalable de terrain. L'objectif de cette première étape est de croiser les regards du gestionnaire d'espaces naturels, de l'écologue et du paysagiste pour optimiser les choix d'observations.

9 Concrètement, l'écologue fournit les cartes des parcelles sous maîtrise foncière ou d'usage (propriété, baux...) et de répartition des milieux. Il présente également ses problématiques de gestionnaire au regard des différents éléments de diagnostic dont il dispose (plan de gestion des haies, inventaires floristiques et faunistiques, hydrologiques, etc.). Le paysagiste propose alors des emplacements sur des fonds IGN (Scan 25 et orthophotographies). Il s'agit d'identifier des points d'observation dégagés, permettant de suivre les évolutions probables de zones de contact ou des limites de site CREN. Les photographies aériennes réalisées entre 1950 et aujourd'hui sont fréquemment utilisées pour alimenter nos échanges sur les dynamiques d'évolution en cours. Certains choix de points d'observation sont guidés par la dimension du grand paysage. Les connaissances internes sur ces paysages (atlas des paysages régionaux, chartes et plans de paysage, ateliers pédagogiques régionaux, etc.) orientent nos choix sur les structures et les éléments de paysage caractéristiques, qui composent la qualité de la trame en place. Lors de la journée de préimplantation sur le site, il est recherché la préinstallation d'un maximum de points de prises de vue.

10 Dans un deuxième temps, le binôme retourne sur le site pour réaliser l'implantation, c'est-à-dire la mise en place de points de prises de vue fixes à un instant to (initial) sur un site ou un territoire donné. Calibrée sur une journée, la vingtaine de prises de vue fait l'objet d'échanges in situ sur l'objectif de la vue (enjeux écologiques et paysagers), la localisation des points d'observation, les choix de cadrage et les scenarii prospectifs d'évolution. Il s'agit de confronter nos intentions avec la réalité du terrain, de vérifier l'intérêt de chaque observatoire et d'en finaliser les réglages techniques. À ce stade encore, les compromis sont fréquents selon l'influence des repères (trépied à proximité d'un arbre isolé, cadrage calé sur un pied de falaise), de la saison d'observation 
(feuillaison, inondabilité, hydromorphie des sols), de l'accessibilité (gués, ornières, propriétés), de la sensibilité écologique (dérangement de nichée, station d'espèce floristique protégée), des usages (présence de troupeaux, escalade, chasse).

11 Le paysagiste prend plusieurs clichés depuis chaque observatoire en faisant varier la focale, le cadrage ou le format. Pendant ce temps, l'écologue photographie à des fins de repérage visuel le lieu d'observation (emplacement du trépied avec l'opérateur en action). Souvent, des panoramas sont réalisés pour conserver un regard élargi sur l'objet observé. Si la focale de $35 \mathrm{~mm}$ est sans doute celle qui restitue le mieux la vision humaine, elle réduit considérablement le champ d'observation. Ces panoramas sont parfois utilisés en tant que "cliché OPP ", mais plus généralement conservés pour contextualiser la photographie. Les informations relatives aux observatoires sont systématiquement recueillies sur une fiche d'implantation. Sur cette base, de retour dans nos locaux, un arbitrage en interne permet de sélectionner la douzaine de photographies « références » et de préciser le parcours ou itinéraire photographiques à respecter pour bénéficier de conditions d'ensoleillement optimales lors des prises de vue. Les fiches d'implantation sont saisies numériquement, validées avec le gestionnaire du site et mises à disposition de l'équipe et elles rejoignent la photothèque de la structure. Chaque observatoire photographique s'organise dans un dossier dédié " Antenne Paysage-OPP-Département-Nom du site-Implantation-date-Numéro-Nom de l'OPP ». Face à la diversité de nos sites d'intervention (marais, vallées, carrières, landes...), le temps de parcours est un facteur limitant le nombre de points d'observation. La prochaine intervention est planifiée conjointement avec l'écologue en fonction du plan de charge et des prévisions d'évolution du site, en particulier, au regard de la gestion envisagée. 
Figure 1. Fiche d'implantation de l'Observatoire photographique $n^{\circ} 01$ du site des Prairies de Longré en Charente (16)

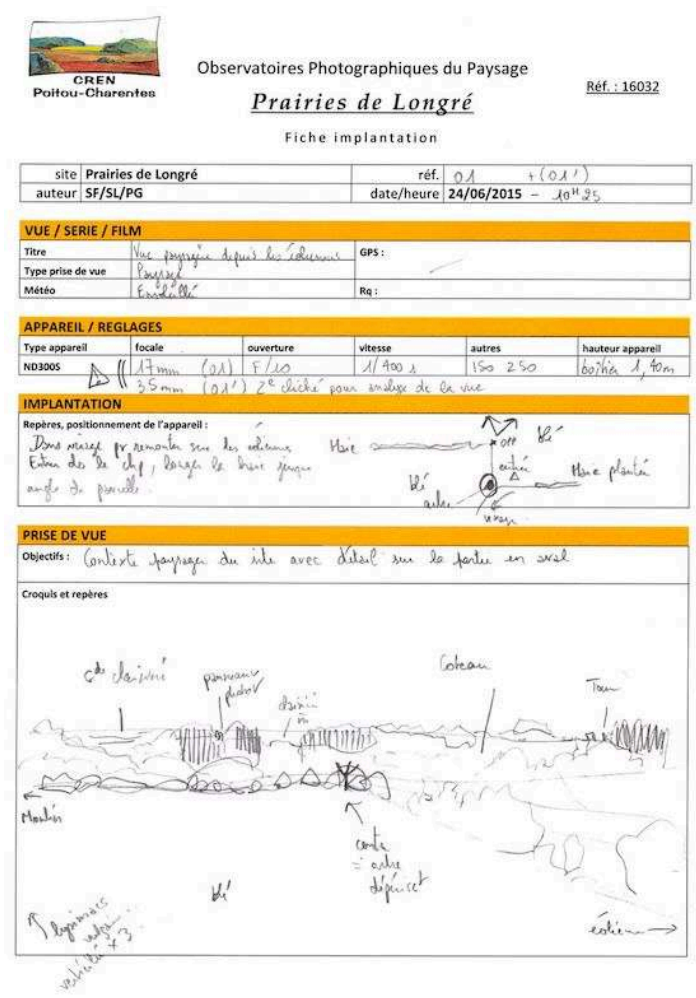

Ce document de terrain permet d'optimiser l'étape suivante (la reconduction), en posant systématiquement le cadre de l'observation (contexte, conditions de prises de vue, objectifs de suivi) Dans l'exemple de ce site de marais charentais, 8 des 12 OPP implantés ont motivé la réalisation de seconds clichés à d'autres focales afin de visualiser le contexte élargi de la prise de vue (le paysage). Ce procédé permet de révéler la complexité d'une trame paysagère (parcellaire laniéré de marais, cloisonné par des boisements), de rendre compte de la distribution des habitats écologiques en fonction de conditions édaphiques particulières (hydromorphie...), ou simplement de prendre du recul.

La troisième étape, dite de «reconduction », consiste à " re-photographier » selon un protocole de prises de vue identiques à celles de l'implantation et à intervalles de temps déterminés $(\mathrm{t} 0+\mathrm{n})$. La difficulté principale réside dans la localisation précise de l'emplacement du point d'observation initial. En effet, revenir sur un site quelques années plus tard rend la tâche ardue et les prouesses technologiques telles que la géolocalisation sont parfois inopérantes. Les fiches d'implantation et la connaissance fine du site par les collaborateurs en charge de la gestion facilitent grandement l'opération. L'interprétation des clichés obtenus entre l'implantation et la reconduction colle au plus près de la réalité du site et s'accompagne de commentaires et de croquis. La phase d'analyse est renouvelée à chaque reconduction. Ces informations sont consignées sur une fiche spécifique. Il arrive régulièrement que les conditions d'observation nécessitent une adaptation (déplacement du point d'observation, cadrage, focale...), c'est le cas notamment lorsqu'un repère fixe disparaît. Il peut s'agir de la suppression d'une clôture, de l'abattage d'un arbre, de la nichée d'une espèce protégée ou d'une crue particulièrement forte.

Lorsqu'on rapproche les prises de vue successives - ou reconductions - effectuées depuis un point $x$ d'observation, on organise ce qu'il est convenu d'appeler une série photographique. Étant donné qu'un site regroupe différents points d'observation, plusieurs séries photographiques sont ainsi directement exploitées pour élaborer ou 
renouveler nos documents de gestion du site, feuille de route des actions à mener pour les 5 à 10 années à venir. Ce document a vocation à formaliser les objectifs et les modalités de gestion, après avoir synthétisé les intérêts et les enjeux du site.

Sans énoncer l'ensemble des données recueillies, le document de gestion compile, dans une partie "présentation", des documents tels que la confrontation d'anciennes photographies aériennes à une actuelle. Chaque domaine est abordé et illustré notamment par des cartes thématiques : géologie, hydrographie, paysages, etc. En ce sens, le document constitue un socle de connaissances commun à l'ensemble des acteurs en lien avec le site (agriculteurs, pêcheurs, chasseurs, randonneurs, habitants...). Outre la description systématique du contexte et de l'intérêt paysager du site - lorsque l'outil "Observatoire photographique » est employé, une fiche-action spécifique est rédigée faisant état des objectifs de l'outil, de la localisation des observatoires et du calendrier prévisionnel de l'outil sur le site concerné. Cette ficheaction est donc associée aux autres telles que des opérations d'entretien ou de reconquête écologique et paysagère (plantations, restauration de mares, de murets en pierres sèches...), actions sur lesquelles l'antenne paysage est également mobilisée (projet, consultation des entreprises, suivi de travaux).

Figure 2. Fiche-action dédiée à l'Observatoire photographique du paysage du site Plaine et marais de Sainte-Soline dans les Deux-Sèvres (79)

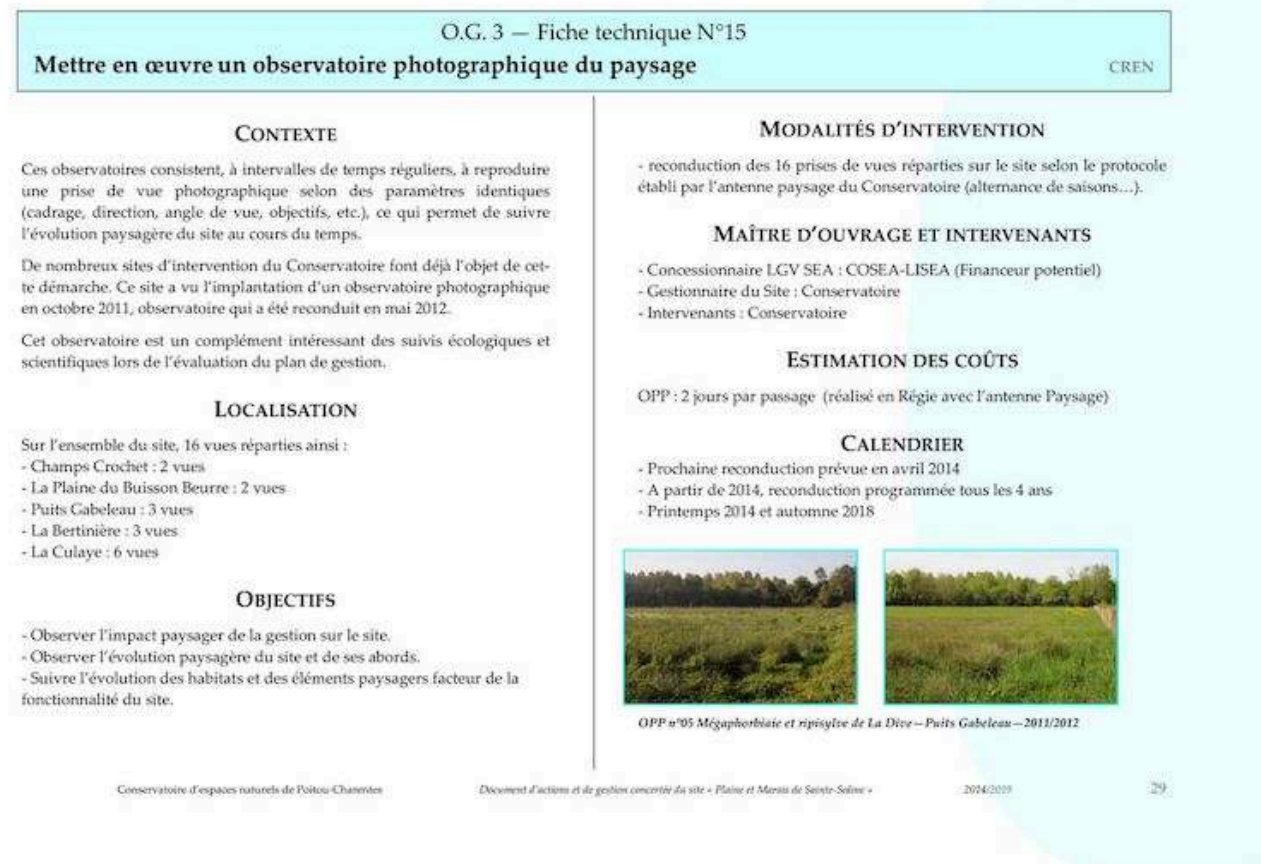

Ce site bénéficie de plusieurs reconductions et d'un plan de gestion. Ici les OPP témoignent des évolutions rapides de 5 secteurs où intervient le CREN tels que les abords d'un fond de vallée, une zone de reconversion en prairies pâturées, anciennement cultivées en maïs ou en peupleraies, le rebord d'un plateau céréalier exploité en agriculture biologique et un îlot alternant pâturage et cultures aux abords du village. De nombreux travaux de gestion sont ainsi visibles à travers les séries photographiques produites : mise en place de clôtures et de troupeaux, plantations de haies, creusement de mares...

$\mathrm{Au}$ même titre que la validation des dossiers d'intervention et de gestion des sites, la méthodologie et la stratégie de développement de l'outil «Observatoires 
photographiques » ont bénéficié de l'expertise du Conseil scientifique et technique (CST) du Conservatoire, constitué de 26 membres spécialisés en sciences de la vie et en techniques de gestion des milieux naturels. Le conseiller-référent «PaysageObservatoires photographiques » du CST est inspecteur des sites au sein de la direction régionale de l'Environnement, de l'Aménagement et du Logement de la NouvelleAquitaine. À bien des égards, il contribue à l'efficience des actions du Conservatoire en faveur des paysages régionaux.

16 Au-delà de l'approche scientifique, l'avancement quantitatif et qualitatif de ce travail est présenté au conseil d'administration du CREN. Les Observatoires photographiques du paysage rendent compte des actions de gestion sur nos sites en lien étroit avec la stratégie d'intervention de la structure. Le suivi photographique des travaux favorise une lecture qualitative des actions réalisées, dépassant ainsi la simple approche quantitative (surface et coût), nécessaire par ailleurs aux financeurs. Pour garantir la lisibilité de nos actions, il peut être engagé pour un site donné la production d'un livret spécifique sur les observatoires. Le document présente les cartographies actuelles de localisation, l'orientation des OPP, le périmètre d'intervention, la liste et les fiches des $\mathrm{OPP}$, et le calendrier des prochaines interventions.

17 La vie d'un site du Conservatoire est également rythmée par des Comités partenariaux de gestion (CPG). Il s'agit de groupes de travail locaux représentés par des partenaires techniques ou financiers et ouverts aux acteurs du territoire considéré. L'outil OPP intervient régulièrement dans cette phase de concertation sur le devenir d'un site, d'un milieu ou d'un paysage. Il permet la transparence de nos actions et facilite la prise de décisions. Le fait que le paysagiste-photographe suive une diversité de sites avec une pluralité de gestionnaires lui permet de se forger une culture environnementale précieuse dans l'exercice de ses autres missions. Pour exemple, dans le cadre d'assistance technique auprès de collectivités porteuses d'une démarche de paysage, l'outil OPP peut démontrer la vitesse d'évolution paysagère sur le territoire, révéler la plus-value paysagère d'une gestion conservatoire et sa durabilité, y compris sur le plan social et économique. De même, les séries photographiques du CREN témoignent fréquemment de la réversibilité des pratiques qui ont perturbé l'écriture paysagère d'un territoire. Les décideurs notamment sont en mesure d'apprécier les orientations possibles en croisant l'ensemble des problématiques (quantité et qualité de l'eau, maintien de l'agriculture, attractivité du territoire, qualité du cadre de vie, ressources).

\section{Cadrer l'outil et avoir conscience de ses limites}

18 Le calibrage de l'outil s'impose et explique en grande partie le choix du Conservatoire à ne pas multiplier les points d'observation, entre raison et représentativité, un exercice auquel les paysagistes-concepteurs sont généralement rompus. En outre, en région, face à l'érosion rapide de la biodiversité, la structure est de plus en plus sollicitée pour intervenir sur les territoires en faveur du patrimoine naturel. Il en résulte une multiplication de sites d'intervention si bien que le fait de cerner les enjeux d'évolution est désormais impératif pour permettre de hiérarchiser l'intérêt de telle implantation par rapport à telle autre. Pour exemple, les sites tels que les cavités à chauve-souris sont peu équipés d'observatoires, à moins que la maîtrise foncière ou d'usage ne soit étendue et qu'ainsi le site participe véritablement aux qualités paysagères du secteur. 
19 De plus, la mémoire d'un lieu et d'un paysage s'oublie vite. De manière générale, les recherches historiques telles que la collecte de témoignages ou de cartes postales anciennes sur nos territoires d'intervention peuvent être améliorées. Stratégiquement, nous priorisons ces recherches dans le cadre de stages de paysage, voire d'ateliers pédagogiques régionaux réalisés avec les écoles nationales supérieures de paysage. Lorsque des photographies anciennes ont pu être retrouvées sur un site donné, la reconduction de telles prises de vue est souvent d'autant plus riche d'enseignement que le pas de temps entre les deux observations est important. C'est l'occasion également de démontrer les dynamiques paysagères à plus vaste échelle. Notons enfin que ces espaces naturels appartiennent à un territoire rural peu photographié par le passé, exception faite des sites pittoresques, élevés au rang de monuments naturels ou de scènes villageoises.

L'outil «Observatoire photographique » permet de réaliser un suivi esthétique et paysager d'un site et de ses abords (ambiances paysagères, aménagement d'un sentier, mise en culture d'une parcelle proche du site...). Cette approche contextuelle, véritable travail d'observation des limites d'un espace naturel avec son environnement immédiat rend compte de la trajectoire paysagère et écologique des espaces gérés. Certains observatoires sont implantés à seule fin de sensibiliser les acteurs locaux aux évolutions de leurs paysages. Un des enjeux des séries photographiques est de nourrir une culture commune de ces espaces et d'en offrir une lecture prospective pour une prise en compte du paysage durable et responsable. Le protocole photographique répond également à notre besoin d'observer, in situ, l'impact paysager de la gestion (curage de fossé, creusement de mare, débardage d'arbres, restauration de prairie...).

La présence de deux paysagistes DPLG au sein de la structure a sensibilisé l'équipe à ces aspects, notamment en identifiant les impacts prévisibles de toute opération de nature à modifier l'aspect paysager d'un site. Lorsque les enjeux paysagers et écologiques le nécessitent, l'outil permet aussi de contribuer au suivi de l'évolution naturelle du site (dynamique de boisement, évolution saisonnière...).

Notons également que la mise en place d'OPP trouve sur son chemin des limites à la fois climatiques, humaines, matérielles, spatiales et temporelles. À l'instar de cette dernière dimension, les rythmes et les facteurs d'évolution de nos paysages diffèrent sensiblement selon les territoires, nécessitant, pour le photographe de paysage, à la fois patience et réactivité en fonction des cas étudiés.

\section{Un travail d'intérêt général qui naturellement se partage}

Dans le cadre de la mise en place de la politique nationale de la trame verte et bleue, et notamment du schéma régional de cohérence écologique, les sites d'intervention du Conservatoire sont majoritairement identifiés en tant que " cœurs de nature ». L'outil «Observatoires photographiques du paysage» approfondit la connaissance de ces enjeux et de ces potentialités de continuités écologiques et paysagères en renseignant et en accompagnant, à son échelle, la qualité paysagère de ces espaces, complétant utilement l'approche cartographique. 
L'exemple d'un enjeu de continuum de pelouses sèches est assez révélateur. À eux seuls, une flèche ou un zonage sur carte ne suffisent pas à qualifier les caractéristiques spatiales de ces secteurs, tandis que les OPP peuvent démontrer leur diversité, voire leur potentialité. Dans cet exemple, le suivi photographique d'un boisement de pente reconverti en pelouses sèches et géré par le Conservatoire témoigne du champ des possibles. Outre la libre circulation des espèces inféodées à ce milieu, la réouverture paysagère se traduit parfois par de belles surprises telles que la présence d'anciens pierriers dévalant les coteaux et favorisant une nouvelle lecture paysagère des lieux (parcellaire, usages, topographie).

Le Conservatoire intervient sur 125 sites pour une surface en maîtrise foncière (acquisition) ou d'usage (baux, conventions) d'environ 10000 hectares. Les espaces naturels témoignent de l'histoire de nos paysages - cultures, pratiques, représentations -, ils constituent des espaces d'intérêt public, accessibles pour bon nombre d'entre eux. Certains sites se prêtent à des expérimentations ou à des actions pédagogiques (écoles, universités, formations, animations et sorties nature), grâce à différentes approches telles que l'écologie, la flore, la faune, la biologie, la géologie et le paysage.

Le dénominateur commun à ce réseau de sites d'intervention gérés et observés réside dans un fonctionnement associant des acteurs locaux et des partenaires impliqués dans la sauvegarde, la protection, la gestion et la valorisation de ce patrimoine naturel commun et sa transmission aux générations futures. L'Observatoire photographique est précieux en tant qu'instrument d'échanges et de débats d'un site ou territoire.

Plus largement, nos Observatoires photographiques du paysage constituent peu à peu un fonds photographique régional dédié aux espaces naturels. Cette production croise les niveaux de lecture de paysage (trames, structures et éléments) à celle de la diversité écologique. À l'image de sa reconnaissance "d'intérêt général », le CREN a vocation à aider le réseau d'acteurs et de partenaires à travers un retour d'expériences et à favoriser les conditions de l'appropriation de ce patrimoine naturel par les citoyens. 
Insérer fig. 3. Extrait d'une double page du livret du CREN sur les Observatoires photographiques du paysage en Poitou-Charentes

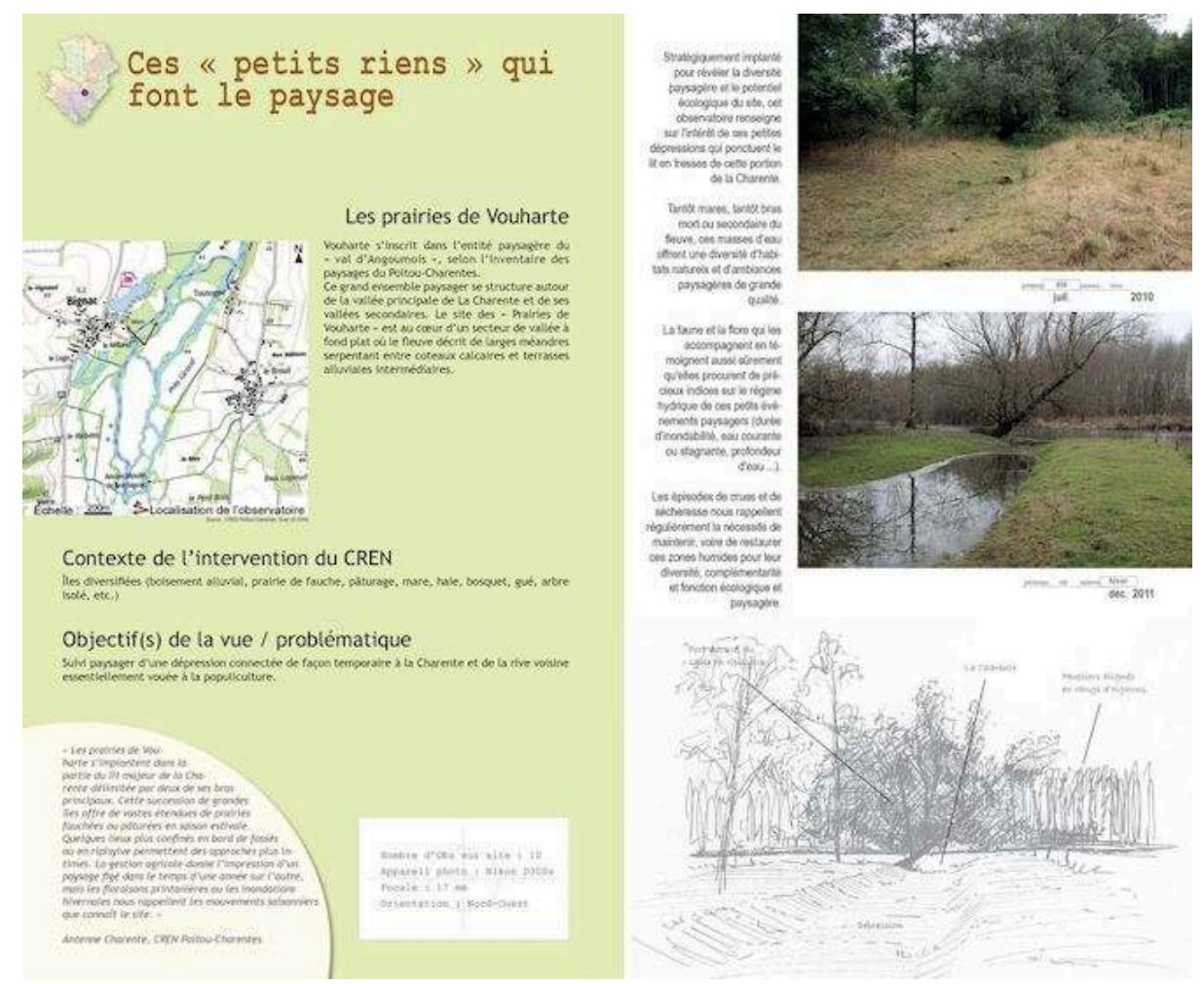

Réalisé avec le soutien de l'État et de la Région, ce document de sensibilisation porte un éclairage sur les Observatoires photographiques du territoire et expose l'utilisation de l'outil par le Conservatoire. Les quelques séries présentées témoignent de l'adaptabilité des OPP à des objectifs et à des problématiques variées, à la condition qu'un cadre rigoureux et pérenne d'observation soit mis en place dès le départ.

Avec la conduite en tant que maître d'ouvrage de l'Atlas des paysages de PoitouCharentes, le CREN est identifié depuis 1997 pour sa compétence "paysage » et très régulièrement sollicité sur le fonctionnement de l'outil. De l'échelle communale à l'échelle régionale, de nombreux acteurs du paysage ont bénéficié d'avis, de conseils, de formations et d'assistances techniques pour, à leur tour, mettre en place des Observatoires photographiques du paysage.

Seul Conservatoire d'espaces naturels (CEN) en France doté d'une antenne " Paysage ", le CREN communique régulièrement au sein du réseau national des CEN sur l'outil. Pour exemple, en 2013, lors d'une réunion de travail du réseau, l'antenne a présenté l'outil, les objectifs, la méthode, les moyens et résultats de la production. Des échanges réguliers se font depuis entre les Conservatoires, notamment sur les conditions de mise en œuvre in situ. À noter, si l'intérêt de l'outil est entendu au sein des autres CEN, leur utilisation se focalise généralement sur la production de courtes séries photographiques, privilégiant d'ailleurs très souvent des sites remarquables sur les plans esthétique et paysager.

31 Face à cet enjeu de "transmission », le Conservatoire de Poitou-Charentes s'impose un protocole de suivi du patrimoine naturel capable d'être aisément appropriable sur les territoires. Outre la nécessaire rigueur de l'outil, retenons qu'il faut apprendre à poser le regard sur le contexte paysager d'un territoire et accompagner le récit de la photographie par des commentaires, des cartographies et des croquis. 


\section{BIBLIOGRAPHIE}

Aubel, C., Bigot, C., Collin, M., Outside Architectes Paysagistes, Minier, J.-P., Inventaire et Atlas des paysages de Poitou-Charentes, 1999.

Conservatoire régional d'espaces naturels de Poitou-Charentes, Poitou-Charentes. Paysages et Nature. Biodiversité, La Crèche, Geste éditions, 2015, 340 p.

Ministère de l'Écologie, de l'Énergie, du Développement durable et de l'Aménagement du territoire, «Itinéraires photographiques. Méthode de l'observatoire photographique du paysage ", 2008, URL : https://www.ecologique-solidaire.gouv.fr/sites/default/files/ M\%C3\%A9thode\%20de\%20l\%27Observatoire\%20photographique\%20du\%20paysage\%20\%202008.pdf.

Séquences Paysages. Revue de l'Observatoire photographique du paysage, Paris, ministère de l'Environnement/Hazan, n 1, 1997.

\section{NOTES}

1. L'itinéraire photographique est un parcours virtuel dans un territoire, qui rend compte des préoccupations des maîtres d'ouvrage en matière de paysage. Le choix des points de vue résulte d'un dialogue entre le photographe et la maîtrise d'ouvrage, assistée par le comité de pilotage, pendant la phase de création de l'itinéraire. Chacun de ces points de vue fait l'objet de rephotographies à des intervalles définis en fonction des dynamiques paysagères à l'œuvre pendant toute la gestion de l'itinéraire photographique. Il est constitué des épreuves des points de vue initiaux, supplémentaires, et des documents associés : éléments d'identification, carnet de route, grille d'analyse, archives (comptes rendus, contrats avec les photographes...).

\section{RÉSUMÉS}

Depuis 1998, le Conservatoire régional d'espaces naturels (CREN) de Poitou-Charentes a développé les Observatoires photographiques du paysage (OPP) pour observer et évaluer spécifiquement l'évolution des espaces naturels où il intervient. Chacun des sites équipés de l'outil regroupe plusieurs points d'observation qui fournissent au total 450 séries photographiques. Ces dernières sont notamment exploitées pour élaborer ou renouveler les documents de gestion et nourrissent peu à peu un fonds photographique régional dédié aux espaces naturels. En Poitou-Charentes, face à l'érosion rapide de la biodiversité, la structure est de plus en plus sollicitée pour intervenir sur les territoires en faveur du patrimoine naturel. Ainsi, de l'échelle communale à l'échelle régionale, de nombreux acteurs du paysage bénéficient d'avis, de conseils, de formations et d'assistances techniques pour, à leur tour, mettre en place des Observatoires photographiques du paysage. 
Since 1994, the Conservatoire régional d'espaces naturels (CREN) of the region of Poitou-Charentes set up Photographic Observatories of the Landscape to observe and monitor changes in the natural spaces of the region. Each site in the programme comprises several observation points supplying a total of 450 series of photographs. These series are used to compile or update planning documents and are added to a regional photographic archive dedicated to natural spaces. In Poitou-Charentes, due to the rapid decline in biodiversity, the CREN is increasingly called on to intervene in support of the conservation of the region's natural heritage. As a result, many stakeholders at the municipal and regional levels benefit from advice as well as technical support and training in setting up their own Photographic Observatories of the Landscape.

\section{INDEX}

Mots-clés : observatoires photographiques du paysage, paysagiste-photographe, suivi photographique, Conservatoire régional d'espaces naturels de Poitou-Charentes, PoitouCharentes

Keywords : Photographic Observatories of the Landscape, landscape architect-photographer, photographic monitoring, The Poitou-Charentes Regional Conservatory of Natural Spaces, Poitou-Charentes

\section{AUTEUR \\ PATRICK GUÉDON}

Paysagiste DPLG, Patrick Guédon est chargé de mission de l'antenne « paysage » du Conservatoire régional d'espaces naturels de Poitou-Charentes. Aux côtés de Jean-Philippe Minier, il contribue à la diffusion de l'Atlas des paysages, aux ateliers des écoles de paysage, appuie les collectivités engagées dans une démarche de projet de paysage (plans de paysage et chartes paysagères). Il accompagne la vie des sites d'intervention du Conservatoire.

guedon[at]cren-poitou-charentes[dot]org

http://www.cren-poitou-charentes.org 